P. Komjáth, Dept. Comp. Sci. Eötvös University, Budapest, Múzeum krt 6-8, 1088, Hungary, e-mail: kope@cs.elte.hu

S. Shelah, Inst. of Mathematics, Hebrew University, Jerusalem, Israel, e-mail: shelah@sunrise.huji.ac.il

\title{
On uniformly antisymmetric functions
}

\section{Introduction}

Recently there has been considerable research on symmetric properties of functions, i.e., when e.g. continuity is replaced by the limit properties of $f(x+h)-f(x-h)(h \rightarrow 0)$. The excellent monograph [6] surveys most of the recent developments.

The following definiton was considered by Evans and Larson (in Santa Barbara, 1984) and by Kostyrko (in Smolenice, 1991).

Definition. A uniformly antisymmetric function is an $f: \mathbf{R} \rightarrow \mathbf{R}$ such that for every $x \in \mathbf{R}$ there is a $d(x)>0$ so that $0<h<d(x)$ implies $|f(x+h)-f(x-h)| \geq d(x)$.

They posed the question if there exists a uniformly antisymmetric function. Kostyrko showed that no such function with a two element range exists, that is, there is no uniformly antisymmetric set (see [5]). This was extended to functions with 3 -element ranges by Ciesielski in [1]. In [2] a uniformly antisymmetric function $f: \mathbf{R} \rightarrow \omega$ was constructed. It had the stronger property that for every $x \in \mathbf{R}$ the set $S_{x}=\{h>0: f(x-h)=f(x+h)\}$ is finite. [2] contains several other relevant results and questions. Kostyrko's result is extended to functions defined on any uncountable subfield of the reals. The authors of [2] ask if this can be extended to countable subfields, as well. As for functions defined on $\mathbf{R}$ they ask if there is an $f: \mathbf{R} \rightarrow \omega$ such that $\left|S_{x}\right| \leq 1$ for $x \in \mathbf{R}$, or if there is an $f$ with finite range that $S_{x}$ is always finite.

In this paper we solve some of those problems. We show that there is always a uniformly antisymmetric $f: A \rightarrow\{0,1\}$ if $A \subset \mathbf{R}$ is countable. We prove that the continuum hypothesis is equivalent to the statement that there is an $f: \mathbf{R} \rightarrow \omega$ with $\left|S_{x}\right| \leq 1$ for every $x \in \mathbf{R}$. If the continuum is at least $\aleph_{n}$ then there exists a point $x$ such that $S_{x}$ has at least $2^{n}-1$ elements. We also show that there is a function $f: \mathbf{Q} \rightarrow\{0,1,2,3\}$ such that $S_{x}$ is always finite, but no such function with finite range on $\mathbf{R}$ exists.

Notation. We use the standard set theory notation. Notably, $\omega$ is the set of natural numbers, ordinals are identified with the sets of smaller ordinals. $\mathbf{R}$ is the set of reals, $\mathbf{Q}$ is the set of rationals. $|A|$ denotes the cardinality of $A$. If $A$ is a set, $\kappa$ is a cardinal, then $[A]^{\kappa}=\{X \subseteq A:|X|=\kappa\},[A]^{<\kappa}=\{X \subseteq A:|X|<\kappa\}$. CH denotes the continuum hypothesis, i.e., that $|\mathbf{R}|=\aleph_{1}$.

No. 502 on the second author's list. Supported by the Hungarian OTKA grant No. 1908 and by the grant of the Israeli Academy of Sciences.

AMS subject classification (1991): 26 A 15, 03 E 50, 04 A 20. 


\section{Uniformly antisymmetric functions on countable sets}

Theorem 1. If $A \subseteq \mathbf{R}$ is countable, then there is a uniformly antisymmetric function $f: A \rightarrow\{0,1\}$.

Proof. Enumerate $A$ as $A=\left\{a_{1}, a_{2}, \ldots\right\}$. By induction on $n<\omega$ we define a finite set $\mathcal{I}_{n}=\left\{I_{\gamma}: \gamma \in \Gamma_{n}\right\}$ of open intervals such that $\emptyset=\Gamma_{0} \subseteq \Gamma_{1} \subseteq \ldots$, so $\emptyset=\mathcal{I}_{0} \subseteq \mathcal{I}_{1} \subseteq \ldots$, each $I_{\gamma}$ is of the form $I_{\gamma}=\left(b_{\gamma}-h_{\gamma}, b_{\gamma}+h_{\gamma}\right)$ with the following properties. Put $B_{n}=$ $\left\{b_{\gamma}: \gamma \in \Gamma_{n}\right\}$.

(1) If $\gamma \neq \gamma^{\prime}$ then either $I_{\gamma} \cap I_{\gamma^{\prime}}=\emptyset$, or one of them contains the other;

(2) if $I_{\gamma^{\prime}} \subseteq I_{\gamma}$ then either $I_{\gamma^{\prime}} \subseteq\left(b_{\gamma}-h_{\gamma}, b_{\gamma}\right)$ or $I_{\gamma^{\prime}} \subseteq\left(b_{\gamma}, b_{\gamma}+h_{\gamma}\right)$;

(3) $\left\{a_{1}, \ldots, a_{n}\right\} \subseteq B_{n}$;

(4) $b_{\gamma} \pm h_{\gamma} \notin A\left(\gamma \in \Gamma_{n}\right)$;

(5) if we put $\varphi_{\gamma}(x)=2 b_{\gamma}-x\left(x \in I_{\gamma}, x \neq b_{\gamma}\right)$, then for $I_{\gamma^{\prime}} \subseteq I_{\gamma}, \varphi_{\gamma}\left(I_{\gamma^{\prime}}\right) \in \mathcal{I}_{n}$ holds.

To start, we put $\Gamma_{0}=\emptyset$.

If $\Gamma_{n-1}$ is already given, and $a_{n} \in B_{n-1}$, put $\Gamma_{n}=\Gamma_{n-1}$. Otherwise, let $I_{\gamma}$ be the unique shortest interval in $\mathcal{I}_{n-1}$ containing $a_{n}$ if there exists one. Select $I=\left(a_{n}-h, a_{n}+h\right)$ in such a way that it is either in $\left(b_{\gamma}-h_{\gamma}, b_{\gamma}\right)$ or in $\left(b_{\gamma}, b_{\gamma}+h_{\gamma}\right)$ and $\varphi_{\gamma_{1}} \cdots \varphi_{\gamma_{r}}\left(a_{n} \pm h\right) \notin A$ for any (applicable) product $\left(\gamma_{i} \in \Gamma_{n-1}\right)$. Notice that the number of those products is $2^{t}$ where $t$ is the number of intervals in $\mathcal{I}_{n-1}$ containing $a_{n}$. Now add all $\varphi_{\gamma_{1}} \cdots \varphi_{\gamma_{r}}(I)$ to $\mathcal{I}_{n-1}$ and get $\mathcal{I}_{n}$. If no interval of $\mathcal{I}_{n-1}$ contains $a_{n}$ then let $I=\left(a_{n}-h, a_{n}+h\right), a_{n} \pm h \notin A$ be an arbitrary interval disjoint from those in $\mathcal{I}_{n-1}$ and add it to get $\mathcal{I}_{n}$.

To conclude the proof of the Theorem we are going to show that there exists a function $f: \mathbf{R} \rightarrow\{0,1\}$ such that $f\left(\varphi_{\gamma}(x)\right)=1-f(x)\left(\gamma \in \bigcup \Gamma_{n}\right)$. As $\varphi_{\gamma}^{2}$ is always a partial identity it suffices to show that no $x \in \mathbf{R}$ is a fixed point of the product of odd many $\varphi_{\gamma}$.

Assume that $x=\varphi_{\gamma_{1}} \varphi_{\gamma_{2}} \cdots \varphi_{\gamma_{t}}(x), t$ odd. Among the intervals $I_{\gamma_{1}}, \ldots, I_{\gamma_{t}}$ there is a longest one, say $I_{\gamma}$ and that must contain all the others. At every appearence of $\varphi_{\gamma}$ in the product $\varphi_{\gamma_{1}} \varphi_{\gamma_{2}} \cdots \varphi_{\gamma_{t}}$ the image of $x$ moves from one side of $b_{\gamma}$ to the other. $\varphi_{\gamma}$ therefore appears even times. In the product the interval $\varphi_{\gamma} \varphi_{\gamma_{i}} \cdots \varphi_{\gamma_{j}} \varphi_{\gamma}$ can be replaced by $\varphi_{\gamma_{i}^{\prime}} \cdots \varphi_{\gamma_{j}^{\prime}}$ where $I_{\gamma_{r}^{\prime}}=\varphi_{\gamma}\left(I_{\gamma_{r}}\right)(i \leq r \leq j)$, so eventually we succeed in eliminating an even number of $\varphi$ 's. We got a shorter formula $x=\varphi_{\gamma_{1}^{\prime}} \cdots \varphi_{\gamma_{t^{\prime}}^{\prime}}(x)$, but $t^{\prime}$ is still odd. Finally we get that $x=\varphi_{\gamma}^{t}(x)$ for some odd $t$ which is impossible.

\section{When $S_{x}$ is finite}

Definition. If $f: \mathbf{R} \rightarrow \omega$ is a function, then for $x \in \mathbf{R}$, set $S_{x}=\{h>0: f(x-h)=$ $f(x+h)\}$.

Theorem 2. There is a function $F:\left[\omega_{1}\right]^{<\omega} \rightarrow \omega$ such that

(a) if $F(A)=F(B)$ then $|A|=|B|$;

(b) if $F(A)=F(B)$ then $A \cap B$ is an initial segment in $A, B$; and

(c) there do not exist $A_{0}, B_{0}, A_{1}, B_{1} \in\left[\omega_{1}\right]^{<\omega}$ such that $A_{0} \cup B_{0}=A_{1} \cup B_{1}, F\left(A_{0}\right)=$ $F\left(B_{0}\right), F\left(A_{1}\right)=F\left(B_{1}\right), A_{0} \neq B_{0}, A_{1} \neq B_{1}$, and $\left\{A_{0}, B_{0}\right\} \neq\left\{A_{1}, B_{1}\right\}$.

Proof. Let the diadic intervals of $\mathbf{R}$ be $I_{0}, I_{1}, \ldots$ For $\alpha<\omega_{1}$ enumerate $\alpha$ as $\alpha=$ $\{\gamma(\alpha, i): i<\omega\}$. (Recall that by our axiomatic set theory assumptions $\alpha$ is identified with the set of smaller ordinals.) Select different irrational numbers $r_{\alpha}$ for $\alpha<\omega_{1}$. We define 
a function $c:\left[\omega_{1}\right]^{2} \rightarrow \omega$ as follows. We construct $c(\beta, \alpha)$ by induction on $\beta$, in the order of the enumeration of $\alpha$. For $\beta<\alpha$, if $\beta=\gamma(\alpha, i)$, let $c(\beta, \alpha)$ be some $j<\omega$ such that

(1) $j>c(\gamma(\alpha, 0), \alpha), \ldots, c(\gamma(\alpha, i-1), \alpha)$;

(2) $r_{\beta} \in I_{j}$

(3) $r_{\alpha} \notin I_{j}$

(4) $r_{\xi} \notin I_{j}$ for $\xi=\gamma(\alpha, 0), \ldots, \gamma(\alpha, i-1)$.

Clearly, such a $j<\omega$ can be found. Let, for $A \in\left[\omega_{1}\right]^{<\omega}, F(A)$ be the isomorphism type of the structure $(A ;<, c)$, i.e., $F(A)=F(B)$ iff $|A|=|B|$ and $c\left(a_{i}, a_{j}\right)=c\left(b_{i}, b_{j}\right)$ whenever $a_{1}<\cdots<a_{n}, b_{1}<\cdots<b_{n}$ are the monotonic enumerations of $A, B$, respectively.

Claim 1. If $F(A)=F(B)$, then $A \cap B$ is an initial segment in both sets.

Proof. Again, let $A=a_{1}, \ldots, a_{n}, B=b_{1}, \ldots, b_{n}$ be the increasing enumerations. Assume that $a_{i}=b_{j}$ is a common element. If $i \neq j$, say $i<j$, then $k=c\left(a_{i}, a_{j}\right)=c\left(b_{i}, b_{j}\right)$ has $r_{a_{i}} \in I_{k}$ (by (2)), and $r_{b_{j}} \notin I_{k}$ (by (3)), a contradiction. So we have that $i=j$. If $t<i$, then, as $c\left(a_{t}, a_{i}\right)=c\left(b_{t}, b_{i}\right)=c\left(b_{t}, a_{i}\right), a_{t}=b_{t}$ by property $(1)$.

Claim 2. There do not exist $\beta, \beta^{\prime}, \alpha, \alpha^{\prime}<\omega_{1} \operatorname{such}$ that $\max \left(\beta, \beta^{\prime}\right)<\min \left(\alpha, \alpha^{\prime}\right), c(\beta, \alpha)=$ $c\left(\beta^{\prime}, \alpha^{\prime}\right)$, and $c\left(\beta^{\prime}, \alpha\right)=c\left(\beta, \alpha^{\prime}\right)$.

Proof. Set $i=c(\beta, \alpha), j=c\left(\beta^{\prime}, \alpha\right)$. As $\beta, \beta^{\prime}<\alpha, i \neq j$, say, $i<j$. Then, considering $c\left(\beta^{\prime}, \alpha\right)$ we get (by (4)) $r_{\beta} \notin I_{j}$ while considering $c\left(\beta, \alpha^{\prime}\right)$ we get that $r_{\beta} \in I_{j}$, a contradiction. If $i>j$ we argue similarly.

Assume now that $F(A)=F(B)$ and we know $A \cup B$. We try to reconstruct $A, B$. Put $X=A \cap B, Y=A-X, Z=B-X$. We can assume that $m^{\prime}=\max (Y)<\max (Z)=m$. In general, to every $x \in Z$ let $x^{\prime}$ be the element in $Y$ corresponding to $x$ under the (unique) order-preserving bijection between $Z$ and $Y$.

For $a<b$ in $A, c(a, b)$ codes a diadic interval including $r_{a}$ but excluding $r_{b}$. The structure $(A ;<, c)$ gives a diadic interval for every element in $A$ separating it from the rest of $A$. As $F(A)=F(B)$ this interval is the same for $x$ and $x^{\prime}$. We get therefore, that there is a diadic interval containing $r_{x}, r_{x^{\prime}}$ but nothing else from $A \cup B$. This makes possible to find $x^{\prime}$ if $x$ is given, or to find $x$ if $x^{\prime}$ is given. Anyway, we can find $m^{\prime}$.

Claim 3. $X=\left\{x \in A \cup B: x<m^{\prime}\right.$ and $\left.c\left(x, m^{\prime}\right)=c(x, m)\right\}$.

Proof. $\subseteq$ is obvious. If, say $x \in Z$ and $c\left(x, m^{\prime}\right)=c(x, m)$ then $c\left(x, m^{\prime}\right)=c(x, m)=$ $c\left(x^{\prime}, m^{\prime}\right)$ a contradiction to $(1)$, as $x \neq x^{\prime}$.

As now $X$ is known, we can decompose $Y \cup Z$ into the pairs $\left\{x, x^{\prime}\right\}$ by the argument before Claim 3. Given such a pair $\{u, v\}$ we have to find if $u \in Z, v \in Y$ or vice versa. We know that $c\left(x^{\prime}, m^{\prime}\right)=c(x, m)$, so, knowing $m, m^{\prime}$ we can identify $x, x^{\prime}$ if we can show that $c\left(x, m^{\prime}\right) \neq c\left(x^{\prime}, m\right)$. But this is done in Claim 2 .

Theorem 3. If $\mathrm{CH}$ holds, then there is a function $f: \mathbf{R} \rightarrow \omega$ such that for every $x \in \mathbf{R}$ $S_{x}$ has at most one element.

Proof. Let $\left\{b_{\alpha}: \alpha<\omega_{1}\right\}$ be a Hamel basis, $F:[\omega]^{<\omega} \rightarrow \omega$ as in Theorem 1. To

$$
x=\sum_{i=1}^{n} \lambda_{i} b_{\alpha_{i}}
$$


$\left(\lambda_{i} \neq 0, \lambda_{i} \in \mathbf{Q}\right), \alpha_{1}<\cdots<\alpha_{n}$ we associate some $f(x)$ that codes the ordered string $\left\langle\lambda_{1}, \ldots, \lambda_{n}\right\rangle$ as well as $F\left(\left\{\alpha_{1}, \ldots, \alpha_{n}\right\}\right)$. This is possible as there are countably many possibilities for both.

Assume that $x \neq y, f(x)=f(y)$. We try to recover the pair $\{x, y\}$ from $x+y$. By our coding of the string of the coefficients in the Hamel basis and the properties of the function $F$ described in the previous Theorem, $x, y$ can be written as

$$
x=\sum_{i=1}^{n} \lambda_{i} b_{\alpha_{i}}, \quad y=\sum_{i=1}^{n} \lambda_{i} b_{\beta_{i}}
$$

such that $\alpha_{i}=\beta_{i}$ for $1 \leq i \leq m$ (some $m<n$ ), and $\left\{\alpha_{m+1}, \ldots, \alpha_{n}\right\} \cap\left\{\beta_{m+1}, \ldots, \beta_{n}\right\}=\emptyset$. $x+y$ can be written in the above basis as

$$
x+y=\sum_{i=1}^{m}\left(2 \lambda_{i}\right) b_{\alpha_{i}}+\sum_{i=m+1}^{n} \lambda_{i} b_{\alpha_{i}}+\sum_{i=m+1}^{n} \lambda_{i} b_{\beta_{i}} .
$$

The support of $x+y$, i.e., the set of those basis vectors in which it has nonzero coefficients is

$$
\left\{\alpha_{1}, \ldots, \alpha_{m}, \alpha_{m+1}, \ldots, \alpha_{n}, \beta_{m+1}, \ldots, \beta_{n}\right\}
$$

from which, by the previous Theorem $\left\{\alpha_{1}, \ldots, \alpha_{n}\right\}$ and $\left\{\beta_{1}, \ldots, \beta_{n}\right\}$ can be recovered. Then we can find $\lambda_{1}, \ldots, \lambda_{n}$, i.e., $x$ and $y$ can be reconstructed.

Before proving that if a vector space $V$ with $|V| \geq \omega_{n}$ is $\omega$-colored then $\left|S_{x}\right| \geq 2^{n}-1$ holds for some $x \in V$ we give a proof of the combinatorial part of the theorem. We then show how to modify the proof to get the stated result.

Theorem 4. If $2 \leq n<\omega$ and $f:\left[\omega_{n}\right]^{<\omega} \rightarrow \omega$ then there exists a set $s \in\left[\omega_{n}\right]^{<\omega}$ which can be written in $2^{n}-1$ ways as the union of two different sets $s=x \cup y$ such that $f(x)=f(y)$.

Proof. Assume that $f:\left[\omega_{n}\right]^{<\omega} \rightarrow \omega$. Select $\omega_{n-1}<y_{n}^{0}<\omega_{n}$ such that it is not in any of the sets

$$
\left\{x: \omega_{n-1}<x<\omega_{n}, f\left(s_{1} \cup\{x\}\right)=j_{1}, \ldots, f\left(s_{t} \cup\{x\}\right)=j_{t}\right\}
$$

(for some $s_{1}, \ldots, s_{t} \in\left[\omega_{n-1}\right]^{<\omega}, j_{1}, \ldots, j_{t}<\omega$ ) which happen to have one element. This is possible, as the number of those sets is $\aleph_{n-1}$, and they are all small enough.

Assume now that $y_{i+1}^{0}, \ldots, y_{n}^{0}$ are already defined. Let $\omega_{i-1}<y_{i}^{0}<\omega_{i}$ be such that it is not in any of the sets of the form

$$
\left\{x: f\left(s_{1} \cup\left\{x, y_{i+1}^{0}, \ldots, y_{n}^{0}\right\}\right)=j_{1}, \ldots, f\left(s_{t} \cup\left\{x, y_{i+1}^{0}, \ldots, y_{n}^{0}\right\}\right)=j_{t}, \omega_{i-1}<x<\omega_{i}\right\}
$$

for some $s_{1}, \ldots, s_{t} \in\left[\omega_{i-1}\right]^{<\omega}, j_{1}, \ldots, j_{t}<\omega$, which are singletons. Again, this choice is possible.

If $y_{1}^{0}, \ldots, y_{n}^{0}$ are given, we define $y_{i}^{1}(1 \leq i \leq n)$ in increasing order. Select $y_{1}^{1} \neq y_{1}^{0}$ such that $\omega<y_{1}^{1}<\omega_{1}$ and $f\left(\left\{y_{1}^{1}, y_{2}^{0}, \ldots, y_{n}^{0}\right\}\right)=f\left(\left\{y_{1}^{0}, \ldots, y_{n}^{0}\right\}\right)$. This is possible, as 
otherwise $y_{1}^{0}$ would be the only element in $\left\{x: \omega<x<\omega_{1}, f\left(\left\{x, y_{2}^{0}, \ldots, y_{n}^{0}\right\}\right)=j\right\}$ where $j=f\left(\left\{y_{1}^{0}, y_{2}^{0}, \ldots, y_{n}^{0}\right\}\right)$, a contradiction to the choice of $y_{1}^{0}$. and

If $y_{1}^{1}, \ldots, y_{i-1}^{1}$ have already been selected, let $y_{i}^{1} \neq y_{i}^{0}$ be such that $\omega_{i-1}<y_{i}^{1}<\omega_{i}$

$$
f\left(s \cup\left\{y_{i}^{1}, y_{i+1}^{0}, \ldots, y_{n}^{0}\right\}\right)=f\left(s \cup\left\{y_{i}^{0}, y_{i+1}^{0}, \ldots, y_{n}^{0}\right\}\right)
$$

for every $s \subseteq\left\{y_{1}^{0}, y_{1}^{1}, \ldots, y_{i-1}^{0}, y_{i-1}^{1}\right\}$. This is possible by the choice of $y_{i}^{0}$.

For $1 \leq k \leq m \leq n, g:\{k, \ldots, m\} \rightarrow\{0,1\}$ put $A=\left\{y_{1}^{0}, y_{1}^{1}, \ldots, y_{k-1}^{0}, y_{k-1}^{1}\right\}$, $B=\left\{y_{k}^{0}, \ldots, y_{m}^{0}\right\}, B^{g}=\left\{y_{k}^{g(k)}, \ldots, y_{m}^{g(m)}\right\}, C=\left\{y_{m+1}^{0}, \ldots, y_{n}^{0}\right\}$.

Claim. $f(A \cup B \cup C)=f\left(A \cup B^{g} \cup C\right)$.

Proof. By induction on $m$. The inductive step trivially follows from the choice of $y_{m}^{1}$.

To conclude the proof of the Theorem, assume that $1 \leq k \leq n, g:\{k, \ldots, n\} \rightarrow\{0,1\}$. Put $A=\left\{y_{1}^{0}, y_{1}^{1}, \ldots, y_{k-1}^{0}, y_{k-1}^{1}\right\}, B^{g}=\left\{y_{k}^{g(k)}, \ldots, y_{n}^{g(n)}\right\}$ and let $1-g$ be the function with $(1-g)(i)=1-g(i)$ for $k \leq i \leq n$. Using the Claim we get that $f\left(A \cup B^{g}\right)=f\left(A \cup B^{1-g}\right)$ and clearly $\left(A \cup B^{g}\right) \cup\left(A \cup B^{1-g}\right)=\left\{y_{1}^{0}, y_{1}^{1}, \ldots, y_{n}^{0}, y_{n}^{1}\right\}$. The number of those decompositions, i.e., that of the pairs $\{g, 1-g\}$ is $2^{n-k}$, summing we get $2^{n-1}+\cdots+1=2^{n}-1$.

Theorem 5. Let $V$ be a vector space, $|V| \geq \aleph_{n}(2 \leq n<\omega)$ and $f: V \rightarrow \omega$ be given. Then $\left|S_{x}\right| \geq 2^{n}-1$ for some $x \in V$.

Proof. Assume that $\left\{b(\alpha): \alpha<\omega_{n}\right\}$ is a linearly independent set. Select $\omega_{n-1}<y_{n}^{0}<\omega_{n}$ outside any of the one-element sets of the form

$$
\begin{aligned}
\left\{\omega_{n-1}<x<\omega_{n}: f\left(\sum_{z \in s_{1}} b(z)+\frac{1}{2} \sum_{z \in s_{1}^{\prime}} b(z)+b(x)\right)\right. & =j_{1}, \ldots, \\
& \left.f\left(\sum_{z \in s_{t}} b(z)+\frac{1}{2} \sum_{z \in s_{t}^{\prime}} b(z)+b(x)\right)=j_{t}\right\}
\end{aligned}
$$

where $s_{1}, s_{1}^{\prime}, \ldots, s_{t}, s_{t}^{\prime} \in\left[\omega_{n-1}\right]^{<\omega}, j_{1} \ldots, j_{t}<\omega$. Given $y_{i+1}^{0}, \ldots, y_{n}^{0}$, let $\omega_{i-1}<y_{i}^{0}<\omega_{i}$ be not in any of the one-element sets

$$
\begin{aligned}
&\left\{\omega_{i-1}<x<\omega_{i}: f\left(\sum_{z \in s_{1}} b(z)\right.\right.\left.+\frac{1}{2} \sum_{z \in s_{1}^{\prime}} b(z)+b(x)+b\left(y_{i+1}^{0}\right)+\cdots+b\left(y_{n}^{0}\right)\right)=j_{1}, \ldots, \\
&\left.f\left(\sum_{z \in s_{t}} b(z)+\frac{1}{2} \sum_{z \in s_{t}^{\prime}} b(z)+b(x)+b\left(y_{i+1}^{0}\right)+\cdots+b\left(y_{n}^{0}\right)\right)=j_{t}\right\}
\end{aligned}
$$

where $s_{1}, s_{1}^{\prime}, \ldots, s_{t}, s_{t}^{\prime} \in\left[\omega_{i-1}\right]^{<\omega}, j_{1}, \ldots, j_{t}<\omega$. If $y_{1}^{0}, \ldots, y_{n}^{0}$ are already constructed, let $y_{1}^{1} \neq y_{1}^{0}$ be such that $\omega<y_{1}^{1}<\omega_{1}$ and $f\left(b\left(y_{1}^{1}\right)+b\left(y_{2}^{0}\right)+\cdots+b\left(y_{n}^{0}\right)\right)=f\left(b\left(y_{1}^{0}\right)+b\left(y_{2}^{0}\right)+\right.$ $\left.\cdots+b\left(y_{n}^{0}\right)\right)$. With $y_{1}^{1}, \ldots, y_{i-1}^{1}$ defined, let $\omega_{i-1}<y_{i}^{1}<\omega_{i}, y_{i}^{1} \neq y_{i}^{0}$ be such that for every $s \cup s^{\prime} \subseteq\left\{y_{1}^{0}, y_{1}^{1}, \ldots, y_{i-1}^{0}, y_{i-1}^{1}\right\}$, if $s \cap s^{\prime}=\emptyset$, then

$$
\begin{aligned}
f\left(\sum_{z \in s} b(z)+\frac{1}{2} \sum_{z \in s^{\prime}} b(z)+b\left(y_{i}^{1}\right)+b\left(y_{i+1}^{0}\right)+\cdots+b\left(y_{n}^{0}\right)\right)= \\
\quad f\left(\sum_{z \in s} b(z)+\frac{1}{2} \sum_{z \in s^{\prime}} b(z)+b\left(y_{i}^{0}\right)+b\left(y_{i+1}^{0}\right)+\cdots+b\left(y_{n}^{0}\right)\right)
\end{aligned}
$$


holds. This is possible by the choice of $y_{i}^{0}$.

For $1 \leq k \leq m \leq n, g:\{k, \ldots, m\} \rightarrow\{0,1\}$ we define

$$
\begin{gathered}
A_{k}=\frac{1}{2}\left(b\left(y_{1}^{0}\right)+b\left(y_{1}^{1}\right)+\cdots+b\left(y_{k-1}^{0}\right)+b\left(y_{k-1}^{1}\right)\right), \\
B=b\left(y_{k}^{0}\right)+\cdots+b\left(y_{m}^{0}\right), \quad B^{g}=b\left(y_{k}^{g(k)}\right)+\cdots+b\left(y_{m}^{g(m)}\right), \\
C=b\left(y_{m+1}^{0}\right)+\cdots+b\left(y_{n}^{0}\right) .
\end{gathered}
$$

Claim. $f\left(A_{k}+B+C\right)=f\left(A_{k}+B^{g}+C\right)$.

Proof. As in Theorem 4.

To conclude the proof one can argue as in Theorem 4 , and decompose $b\left(y_{1}^{0}\right)+b\left(y_{1}^{1}\right)+$ $\cdots+b\left(y_{n}^{0}\right)+b\left(y_{n}^{1}\right)$ in $2^{n}-1$ ways into the sum of two vectors with the same $f$ value as $\left(A_{k}+B^{g}\right)+\left(A_{k}+B^{1-g}\right)$ where $g:\{k, \ldots, n\} \rightarrow\{0,1\}$.

\section{Finite range}

Theorem 6. There is a function $f: \mathbf{Q} \rightarrow\{0,1,2,3\}$ such that for every $x \in \mathbf{Q}, S_{x}$ is finite.

Proof. It suffices to find such a function assuming two values on the set $\mathbf{Q}^{+}=\{x \in$ $\mathbf{Q}: x>0\}$. We decompose $\mathbf{Q}^{+}$into the increasing union of finite sets $A_{1} \subseteq A_{2} \subseteq \cdots$. We also define an auxiliary graph $G$ on $\mathbf{Q}^{+}$. Two points $x$ and $y$ will be joined in $G$ if $(x+y) / 2 \in A_{n}$ for some $n$ but $x, y \notin A_{n+1}$. If, with an appropriate choice of the sets we can guarantee that the graph $G$ is bipartite, then the bipartition of $G$ will give a good function on $\mathbf{Q}^{+}$. Indeed, if $x \in A_{n}$ and $f(x-h)=f(x+h)$ then one of $x-h, x+h$ is in $A_{n+1}$ so there are only finitely many such $h$ 's.

Let a positive rational number be in $A_{n}$ if it is of the form $x=p / n$ ! and $x<2^{n}$. Clearly these sets are finite, constitute an increasing sequence, and their union is $\mathbf{Q}^{+}$.

We first show that if $x, y$ are joined in $G$, then they first appear in the same $A_{n}$. Assume that $x \in A_{n+1}-A_{n}, y \in A_{m+1}-A_{m}, m \geq n$, and $z=(x+y) / 2 \in A_{n-1}$. Then, the denominator of $y=2 z-x$ is (a divisor of) $(n+1)$ !. Also, $y<2 z<2^{n+1}$, so $y \in A_{n+1}$, i.e., $m=n$.

Finally, we show that $G$ on $A_{n+1}-A_{n}$ does not contain odd circuits. Assume that $a_{1}, \ldots, a_{2 u+1}$ is one, i.e., $a_{i}+a_{i+1}=2 b_{i}$ for some $b_{i} \in A_{n-1}(1 \leq i \leq 2 u+1)$. Here, we use cyclical indexing, i.e., $a_{2 u+2}=a_{1}$. Then again, $a_{1}<2 b_{1}<2^{n}$, and as $a_{1}=$ $b_{1}-b_{2}+b_{3}-\cdots+b_{2 u+1}, a_{1}$ has denominator $(n-1)$ !, so it is in $A_{n}$, a contradiction.

Theorem 7. If $f: \mathbf{R} \rightarrow\{1, \ldots, n\}$ is a function, then $S_{x}$ is infinite for some $x \in \mathbf{R}$.

Proof. Actually the result is true for any uncountable vector space $V$ over $\mathbf{Q}$. Assume that $f: V \rightarrow\{1, \ldots, n\}$. Let $\left\{b(\alpha): \alpha<\omega_{1}\right\}$ be linearly independent. For $\beta<\alpha<\omega_{1}$, the formula $F(\beta, \alpha)=f(b(\alpha)-b(\beta))$ defines an $n$-coloring of $\left[\omega_{1}\right]^{2}$. By an old ErdösRado theorem (see Cor. 1, p. 459 in [3]), there are a color $1 \leq k \leq n$ and ordinals $\alpha(0)<\cdots<\alpha(\omega)$, such that $F(\alpha(i), \alpha(j))=k$ for $i<j \leq \omega$. But then,

$$
f(b(\alpha(i))-b(\alpha(0)))=f(b(\alpha(\omega))-b(\alpha(i)))=k,
$$

i.e., the vector $b(\alpha(\omega))-b(\alpha(0))$ can be written infinitely many ways as the sum of two monocolored vectors. 


\section{References}

[1] K. Ciesielski: Notes on problem 1 from "Uniformly antisymmetric functions", to appear.

[2] K. Ciesielski, L. Larson: Uniformly antisymmetric functions, to appear.

[3] P. Erdős, R. Rado: A partition calculus in set theory, Bull. of the Amer. Math. Soc. 62 (1956), 427-489.

[4] P. Komjáth: Vector sets with no repeated differences, Coll. Math. 64(1993), 129-134.

[5] P. Kostyrko: There is no strongly locally antisymmetric set, Real Analysis Exchange, 17 (1991/92), 423-425.

[6] B. S. Thomson: Symmetric properties of real functions, to appear. 